\title{
Numerical simulation of precipitation in multicomponent Ni-base alloys
}

\author{
Luc Rougier ${ }^{\mathrm{a}, \mathrm{b}}$, Alain Jacot ${ }^{\mathrm{a}, *}$, Charles-André Gandin ${ }^{\mathrm{c}}$, Paolo Di Napoli ${ }^{\mathrm{a}}$, \\ Pierre-Yvan Théry ${ }^{b}$, Damien Ponsen ${ }^{b}$, Virginie Jaquet ${ }^{b}$ \\ ${ }^{a}$ Ecole Polytechnique Fédérale de Lausanne, LSMX, MXG, 1015 Lausanne, Switzerland \\ ${ }^{\mathrm{b}}$ Snecma-SAFRAN Group, Service YQGC, 92702 Colombes, France \\ ${ }^{\mathrm{c}}$ MINES ParisTech, CEMEF UMR CNRS 7635, 06904 Sophia Antipolis, France
}

Received 19 December 2012; received in revised form 1 July 2013; accepted 12 July 2013

Available online 12 August 2013

\begin{abstract}
A comprehensive particle size distribution model has been developed for the simulation of $\gamma^{\prime}$ precipitation in multicomponent Ni alloys. Nucleation, growth and coarsening of the precipitates are described by a particle size distribution. The growth rate of each precipitate class is calculated with a multi-component diffusion model formulated for non-diagonal matrices of diffusion coefficients. The model is fully coupled with CALPHAD calculations of the thermodynamic equilibrium at the interface, including a direct treatment of the effect of curvature through modification of the Gibbs free energy. An optimization strategy was developed to minimize the computational cost. The model was used to simulate ageing heat treatment at $600{ }^{\circ} \mathrm{C}$ of $\mathrm{Ni}-7.56$ at. $\%$ Al- 8.56 at. $\% \mathrm{Cr}$, which was studied experimentally by Booth-Morrison and others (Booth-Morrison C, Weninger J, Sudbrack CK, Mao Z, Noebe RD, Seidman DN. Acta Mater 2008;56:3422; Mao Z, Booth-Morrison C, Sudbrack CK, Martin G, Seidman DN. Acta Mater 2012;60:1871). The comparisons showed that the precipitation stages of $\gamma^{\prime}$ precipitates are correctly captured by the numerical model. It was shown that non-diagonal diffusion coefficients substantially influence the selection of the operating tie-line and the overall transformation kinetics. With non-diagonal diffusion matrices, complex phenomena such as uphill diffusion of $\mathrm{Cr}$ due to the $\mathrm{Al}$ gradients were evidenced and explained.

(C) 2013 Acta Materialia Inc. Published by Elsevier Ltd. All rights reserved.
\end{abstract}

Keywords: Precipitation; Superalloys; Modelling; Heat treatment; CALPHAD

\section{Introduction}

Nickel-base superalloys are widely used in the aerospace industry for their outstanding mechanical properties at high temperatures [1]. Their creep resistance depends on the volume fraction and average size of the $\gamma^{\prime}$ precipitates formed in the $\gamma$ matrix. Over the last two decades considerable effort has been made to develop numerical models for the description of precipitation in metallic systems. A first approach is to calculate the evolution of the average radius of the precipitates as a result of Ostwald ripening [2]. The mean radius approach proposed by Langer and Schwarz also includes the nucleation and growth regimes that precede and partly overlap with the coarsening regime [3]. This

\footnotetext{
* Corresponding author. Tel.: +41 21693 3920; fax: +41 216935890 .

E-mail address: alain.jacot@epfl.ch (A. Jacot).
}

approach was limited to a dilute binary alloy and suffers from limitations in the case of complex nucleation sequences, in particular for non-isothermal heat treatments, which can lead to complex particle size distributions (PSDs). For this reason, precipitation models are preferentially based on a direct representation of the PSD, following the numerical model initially proposed by Kampmann and Wagner [4]. The nucleation rate is calculated from the homogeneous or heterogeneous nucleation theory and the concept of critical nucleation radius. Specific examples of the PSD approach are found in Refs. [5-8]. Several models address the problem of precipitation in multicomponent alloys by using the extremum principle and the mean-field approach $[9,10]$. Other modelling approaches are based on the Monte Carlo technique [11] and the cluster dynamic method [12], or simply on the integration of Time Temperature Transformation (TTT) diagrams [13]. These models 
are aimed at being coupled with related additional microstructural features [14] and prediction of mechanical properties [15]. Some of these models have been coupled with the CALPHAD approach $[8,16,17]$.

Modelling precipitation in Ni-base superalloys implies several difficulties. First, the alloy chemistry is very complex, with a large number of alloying elements. For a quantitative prediction of the precipitation kinetics, a multicomponent formulation is thus required and the model should be directly or indirectly coupled with CALPHAD calculations in order to compute the thermodynamic equilibrium. Another difficulty is related to the final volume fraction of precipitates in Ni-base superalloys, which is very large $(60 \%$ or more). Due to the high supersaturations, some of the usual assumptions regarding the growth kinetics are no longer valid [8]. The role of the cross-diffusion, i.e. non-diagonal matrices of diffusion coefficients, is also known to be important in Ni-base alloys [18] and should not be neglected. While individual solutions to these problems already exist, they are seldom combined. Moreover, another difficulty in addressing this problem is to keep a good computational efficiency, in particular when the model is coupled to external CALPHAD computation modules.

In this paper, we present a comprehensive PSD model which addresses the aforementioned difficulties. A particular emphasis is put on the coupling with the CALPHAD approach and the numerical aspects associated with it. The model is applied to a ternary alloy of the $\mathrm{Ni}-\mathrm{Al}-\mathrm{Cr}$ system for which detailed experimental data have been published [19].

\section{Modeling}

The model is based on the discretization of the particle size distribution into size classes. Each class $k$ corresponds to a category of precipitates, having its own values of precipitation variables such as the radius, the number density, the interface and average concentrations. Using the terminology introduced by Perez [20], a so-called Lagrange-like approach is used to track the PSD. As opposed to the Eulerian approach, where a class represents a fixed size interval and particle fluxes between adjacent classes are calculated to describe the evolution of the PSD [21], the Lagrange-like approach consists of tracking the evolution of the precipitation variables of a series of classes of precipitates whose population is normally fixed. Each class represents a family of particles that have nucleated within the same time interval and are assumed to evolve in the same way. The population in a given class is normally kept constant until the class potentially disappears due to the dissolution phenomena.

\subsection{Nucleation}

The nucleation rate of the precipitates, $I\left(\mathrm{~m}^{-3} \mathrm{~s}^{-1}\right)$, is calculated with classical nucleation theory $(\mathrm{CNT})[22,23]$ :
$I=n_{0}\left(1-g^{\gamma^{\prime}}\right) Z \beta e^{-\frac{\Delta G^{*}}{\hbar_{B} T}}\left(1-e^{-\frac{t}{\tau}}\right)$

where $n_{0}$ is the density of potential nucleation sites $\left(\mathrm{m}^{-3}\right)$, $g^{\gamma^{\prime}}$ is the volume fraction of precipitates, $Z=\left(V_{m}^{\gamma^{\prime}} \Delta G_{\text {nucl }}^{\gamma^{\prime}}\right) /\left(8 \pi \sqrt{\left(\sigma^{\gamma / \gamma^{\prime}}\right)^{3} k_{B} T}\right)$ is the Zeldovich factor, $\beta=\left(4 \pi\left(R^{*}\right)^{2} / a^{4}\right) \min _{i}\left(\mid D_{i i}^{\gamma} / \bar{X}_{i}^{\gamma}\right)$ is the rate of attachment of solute atoms on a critical precipitate $\left(\mathrm{s}^{-1}\right), k_{B}$ is the Boltzmann constant $\left(\mathrm{J} \mathrm{K}^{-1}\right), T$ is the temperature $(\mathrm{K}), t$ is the time (s), $a$ is the lattice parameter $(\mathrm{m}), \tau=2 /\left(\pi \beta Z^{2}\right)$ is the incubation time (s), $\sigma^{\gamma / \gamma^{\prime}}$ is the $\gamma / \gamma^{\prime}$ interfacial energy $\left(\mathrm{J} \mathrm{m}^{-2}\right), \Delta G_{\text {nucl }}^{\gamma^{\prime}}$ is the chemical driving force $\left(\mathrm{J} \mathrm{m}^{-3}\right)$ for the nucleation of a critical spherical precipitate of radius $R^{*}$ (m) and $V_{m}^{\gamma^{\prime}}$ is the molar volume of the $\gamma^{\prime}$ phase $\left(\mathrm{m}^{3} \mathrm{~mol}^{-1}\right)$, assumed to be equal to the molar volume of the parent phase $\gamma . D_{i i}^{\gamma}$ and $\bar{X}_{i}^{\gamma}$ are, respectively, the diagonal diffusion coefficient $\left(\mathrm{m}^{2} \mathrm{~s}^{-1}\right)$ and the average concentration in $\gamma$, for the solute elementi. The nucleation barrier, $\Delta G^{*}(\mathrm{~J})$, is given by:

$\Delta G^{*}=\frac{16 \pi}{3} \frac{\left(\sigma^{\gamma / \gamma^{\prime}}\right)^{3}}{\left(\Delta G_{n u c l}^{\gamma^{\prime}}\right)^{2}}$

Nuclei are considered to be stable only beyond an effective radius, $R_{\text {effective, }}$ which is larger than the critical radius from the CNT, $R^{*}$. This hypothesis allows accounting for the size fluctuations of the critical precipitates [20,24]:

$R_{\text {effective }}=R^{*}+\frac{1}{2} \sqrt{\frac{k_{B} T}{\pi \sigma^{\gamma / \gamma^{\prime}}}}$

with

$R^{*}=-\frac{2 \sigma^{\gamma / \gamma^{\prime}}}{\Delta G_{n u c l}^{\gamma^{\prime}}}$

A new PSD class is created if the density of precipitates that have nucleated since the creation of the last class, $n_{\text {cumul }}$, is larger than a user-defined minimum number of particles in a class, $n_{\min }$. This condition expresses mathematically as:

$n_{\text {cumul }}=\int_{t_{\text {last }}}^{t} I(t) d t>n_{\text {min }}$

where $t_{\text {last }}$ is the time of the last class creation event.

If $n_{\text {cumul }}$ is larger than a user-defined maximum number, $n_{\max }$, the time-step is reduced in order to limit the number of particles in a class. The choice of $n_{\min }$ and $n_{\max }$ determines the accuracy of the PSD discretization. Typical values for the calculations presented hereafter are $10^{19}$ and $10^{22} \mathrm{~m}^{-3}$, respectively.

\subsection{Growth/coarsening}

The growth rate is calculated in each class as a function of the current radius, the interface concentrations and the average concentrations of the matrix. Following a mean field approximation, the average concentrations of the matrix are assumed to be common for all classes. 
Neglecting the gradients in the $\gamma^{\prime}$ phase and assuming equal molar volumes in $\gamma$ and $\gamma^{\prime}$ for all elements, the growth rate in a given class $k$ of radius $R_{k}$ must satisfy the following local solute balances:

$v_{k}\left(X_{i, k}^{\gamma^{\prime *}}-X_{i, k}^{\gamma *}\right)+J_{i, k}^{\gamma *}=0 \quad$ for $i=1, \ldots, N$

where $N$ is the number of solute elements, and $X_{i, k}^{\gamma *}$ and $X_{i, k}^{\gamma^{\prime *}}$ are the atomic fractions of element $i$ in class $k$ at the interface in $\gamma$ and $\gamma^{\prime}$, respectively.

The solute flux of element $i$ in $\gamma$ at the $\gamma / \gamma^{\prime}$ interface in class $k$ is obtained from the effective diffusion matrix in the $\gamma$ phase, $D_{i j}^{\gamma}$, and the concentration gradients at the interface:

$J_{i, k}^{* *}=-\left.\sum_{j=1}^{N} D_{i j}^{\gamma} \frac{\partial X_{j, k}^{\gamma}}{\partial r}\right|_{r=R_{k}} \quad$ for $i=1, \ldots, N$

The concentration gradient of element $j$ in $\gamma$ near the $\gamma / \gamma^{\prime}$ interface in class $k$ is estimated with the following expression:

$\left.\frac{\partial X_{j, k}^{\gamma}}{\partial r}\right|_{r=R_{k}}=\frac{\bar{X}_{j}^{\gamma}-X_{j, k}^{\gamma *}}{\delta_{j, k}} \quad$ for $i=1, \ldots, N$

where $\delta_{i, k}$ is the length of the diffusion layer around the precipitates of class $k$ for the solute element $i$. It is linked to the supersaturation $\Omega_{i, k}=\left(\bar{X}_{i}^{\gamma}-X_{i, k}^{\gamma *}\right) /\left(X_{i, k}^{\gamma^{\prime *}}-X_{i, k}^{\gamma *}\right)$ with the exact analytical solution of the diffusion problem in a semiinfinite matrix for a binary alloy $[8,25]$ :

$\delta_{i, k}=\frac{\Omega_{i, k}}{2 \lambda_{i, k}^{2}} R_{k} \quad$ for $i=1, \ldots, N$

where $\lambda_{i, k}$ is defined by the following equation:

$2 \lambda_{i, k}^{2}-2 \lambda_{i, k}^{3} \sqrt{\pi} \exp \left(\lambda_{i, k}^{2}\right) \operatorname{erfc}\left(\lambda_{i, k}\right)=\Omega_{i, k} \quad$ for $i=1, \ldots, N$

Assuming that the interface concentrations are given by the local equilibrium corrected for the Gibbs-Thomson effect, the growth rate of a precipitate class $k$ can be calculated by solving the following set of non-linear equations:

$v_{k}\left(X_{i, k}^{\gamma^{* *}}-X_{i, k}^{\gamma *}\right)=\sum_{j=1}^{N} D_{i j}^{\gamma} \frac{\bar{X}_{j}^{\gamma}-X_{j, k}^{\gamma *}}{\delta_{j, k}} \quad$ for $i=1, \ldots, N$

$X_{i, k}^{\gamma^{\prime *}}=k_{i, k}\left(T, R_{k}, X_{1, k}^{\gamma *}, \ldots, X_{N, k}^{\gamma *}\right) X_{i, k}^{\gamma *} \quad$ for $i=1, \ldots, N$

$T=T_{\text {solvus }}\left(R_{k}, X_{1, k}^{\gamma *}, \ldots, X_{N, k}^{\gamma *}\right)$

where $k_{i, k}$ are the partition coefficients defining the tie-lines and $T_{\text {solvus }}$ is a function representing the solvus temperature. These quantities depend on the precipitate radius due to the Gibbs-Thomson effect. If the functions $k_{i, k}$ and $T_{\text {solvus }}$ are described by a CALPHAD approach, the Gibbs-Thomson effect can be taken into account by adding the following contribution to the Gibbs free energy of the $\gamma^{\prime}$ phase:

$\Delta G_{G T, k}^{\gamma^{\prime}}=\frac{2 \sigma^{\gamma / \gamma^{\prime}} V_{m}^{\gamma^{\prime}}}{R_{k}}$
The model assumes equal mole and volume fractions of phases and elements. At a given temperature $T$, Eqs. (11)(13) form a set of $2 N+1$ equations which can be solved directly to obtain the $2 \mathrm{~N}$ unknown interface concentrations, $X_{i, k}^{\gamma *}$ and $X_{i, k}^{\gamma^{\prime *}}$, and the unknown interface velocity, $v_{k}$. This problem is, however, non-linear and difficult to solve directly if the phase diagram information is obtained from the CALPHAD approach. The chosen strategy is to decompose the problem into a kinetic step and an equilibrium step, during which the local equilibrium at the interface is calculated, taking into account the GibbsThomson effect. The method consists of defining a spherical volume (or cell) around the precipitate/matrix interface, as schematized in Fig. 1. This cell is assumed to be at local thermodynamic equilibrium (with the interface curvature contribution). The volume of $\gamma^{\prime}$ considered in the cell depends on the assumption made for the diffusion in the precipitates. If diffusion is neglected in $\gamma^{\prime}$, the thickness of $\gamma^{\prime}$ embedded in the spherical shell, $h_{k}^{\gamma^{\prime}}$, is equal to the thickness of $\gamma, h_{k}^{\gamma}$, which is taken to be proportional to the minimum depth of the diffusion layers in $\gamma$ defined by the smallest $\delta_{i, k}$ value (Eq. (9)). If complete mixing is assumed in $\gamma^{\prime}$, the whole precipitate is embedded in the cell and $h_{k}^{\gamma^{\prime}}=R_{k}$. This assumption offers the advantages of a simpler formulation and a higher numerical stability, together with a lower computational cost.

The average concentrations in the cell are first calculated from the equilibrium concentrations and the local phase fractions at the previous time-step ( $t$ superscript):

$\bar{X}_{i, k}^{\text {cell }, t}=\varphi_{k}^{\gamma^{\prime}, t} X_{i, k}^{\gamma^{\prime *}, t}+\left(1-\varphi_{k}^{\gamma^{\prime}, t}\right) X_{i, k}^{\gamma *, t}$

where $\varphi_{k}^{\gamma^{\prime}, t}$ is the volume fraction of $\gamma^{\prime}$ in the cell. These quantities are then updated based on the solute fluxes at the interface:

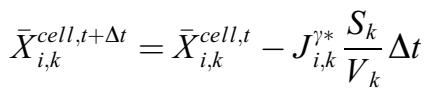

where $\Delta \mathrm{t}$ is the time step, Sk is the outer surface of the precipitate, $\mathrm{Vk}$ is the volume of the cell and $J_{i, k}^{\gamma *}$ is the flux obtained from Eqs. (7)-(10).

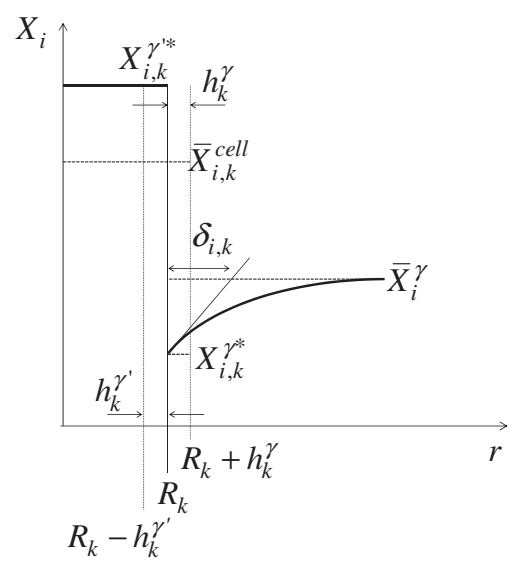

Fig. 1. Schematic concentration fields around the $\gamma^{\prime}$ spherical precipitate $/ \gamma$ matrix interface for class $k$, and the cell representation used to compute local equilibrium. 
The new average concentrations in the cell are then used as a condition to compute the local equilibrium with a call to the software Thermo-Calc through the TQ interface [26], or, alternatively, with an optimization method which will be described in the next paragraph.

The equilibrium calculation provides a new volume fraction of $\gamma^{\prime}$ in the cell, $\varphi_{k}^{\gamma^{\prime}, t+\Delta t}$, which can be converted into a new $\gamma^{\prime}$ radius:

$R_{k}^{t+\Delta t}=\sqrt[3]{\varphi_{k}^{\gamma^{\prime}, t+\Delta t}\left[\left(R_{k}^{t}+h_{k}^{\gamma}\right)^{3}-\left(R_{k}^{t}-h_{k}^{\gamma^{\prime}}\right)^{3}\right]+\left(R_{k}^{t}-h_{k}^{\gamma^{\prime}}\right)^{3}}$

and thus to a radius increment $\Delta R=R_{k}^{t+\Delta t}-R_{k}^{t}$ and a growth rate $v_{k}=\Delta R / \Delta t$.

At each time step, average quantities for the population of the $\gamma^{\prime}$ precipitates such as the total number density, $n_{t o t}$, the fraction of $\gamma^{\prime}$ phase, $g^{\gamma^{\prime}}$, the number averaged radius, $\bar{R}_{n}$, and the fraction averaged radius, $\bar{R}$, are calculated as follows (the time step superscript is omitted for simplicity):

$n_{\text {tot }}=\sum_{k} n_{k}$

$g^{\gamma^{\prime}}=\frac{4 \pi}{3} \sum_{k}\left(n_{k} R_{k}^{3}\right)$

$\bar{R}_{n}=\frac{1}{n_{\text {tot }}} \sum_{k} n_{k} R_{k}$

$\bar{R}=\sqrt[3]{\frac{3 g^{\gamma^{\prime}}}{4 \pi n_{\text {tot }}}}$

The average concentration of the matrix, $\bar{X}_{i}^{\gamma}$, is deduced from a global solute balance:

$\bar{X}_{i}^{\gamma}=\frac{X_{0, i}-g^{\gamma^{\prime}} \bar{X}_{i}^{\gamma^{\prime}}}{1-g^{\gamma^{\prime}}}$

where $X_{0, i}$ is the alloy nominal concentration of solute element $i$ and $\bar{X}_{i}^{\gamma^{\prime}}$ is the average concentration in the precipitates, calculated as follows:

$\bar{X}_{i}^{\gamma^{\prime}}=\frac{\sum_{k} n_{k} R_{k}^{3} \bar{X}_{i, k}^{\gamma^{\prime}}}{\sum_{k} n_{k} R_{k}^{3}}$

where $\bar{X}_{i, k}^{\gamma^{\prime}}$ is the average concentration of solute element $i$ in $\gamma^{\prime}$ for class $k$.

If a uniform concentration profile is assumed in $\gamma^{\prime}, \bar{X}_{i, k}^{\gamma^{\prime}}$ is directly given by the interface concentration $X_{i, k}^{\gamma^{\prime} *}$ (full mixing assumption in the $\gamma^{\prime}$ phase). If the diffusion coefficients are assumed to be 0 in $\gamma^{\prime}$, the average concentrations of the precipitates are calculated from the differences of interface concentrations and volumes between two time steps in the case of growth. In the case of dissolution $\bar{X}_{i}^{\gamma^{\prime}}$ is assumed to remain constant in the class. To represent the PSD, the density of the distribution in a given class $k, D_{k}\left(\mathrm{~m}^{-4}\right)$, is given by the ratio of the precipitate number density, $n_{k}$ $\left(\mathrm{m}^{-3}\right)$, to the size interval:

$D_{k}=\frac{n_{k}}{R_{k+1}-R_{k}}$ where $R_{k}$ and $R_{k+1}$ are the radii of two consecutive classes of the PSD. The cumulative size distribution up to class $k$ is calculated as follows:

$n_{k}^{\text {cum }}=\sum_{i=0}^{k-1} n_{i}+n_{k} \frac{R_{k}-R_{k-1}}{R_{k+1}-R_{k-1}}$

The normalized distribution, $D_{k}^{\text {norm }}$, is calculated using the values of $D_{k}, \bar{R}_{n}$ and $n_{t o t}$ :

$D_{k}^{n o r m}=D_{k} \frac{\bar{R}_{n}}{n_{\text {tot }}}$

$D_{k}^{n o r m}$ is generally represented as a function of the normalized radius, $\rho_{k}$, which is defined by:

$\rho_{k}=\frac{R_{k}}{\bar{R}_{n}}$

During the last stages of precipitation, the number of size classes quickly decreases due to coarsening. In order to keep a smooth evolution of the average quantities, new classes are inserted following the method described by Perez et al. [20].

\subsection{Optimized CALPHAD coupling}

In order to increase the calculation speed, the number of equilibrium calculations performed with the software Thermo-Calc can be substantially reduced by using simplified phase diagrams for the calculation of interface concentrations and phase fractions. For given values of the average concentrations and curvature energy, $\Delta G_{G T}^{\gamma^{\prime}}$, the $\gamma$ solvus is approximated for each size class by the following equation:

$$
\begin{aligned}
T_{\text {solvus }}\left(R_{k}, X_{1, k}^{\gamma *}, \ldots, X_{N, k}^{\gamma *}\right) \cong & T_{\text {solvus }, k}^{r e f}+\sum_{i=1}^{N} a_{i, k}\left(X_{i, k}^{\gamma *}-X_{i, k}^{\gamma * \text { ref }}\right) \\
& +b_{k}\left(\Delta G_{G T, k}^{\gamma^{\prime}}-\Delta G_{G T, k}^{\gamma^{\prime}, r e f}\right)
\end{aligned}
$$

where $a_{i, k}$ is the solvus slope with respect to the concentration of solute element $i$, and $b_{k}$ is the sensitivity coefficient with respect to the curvature energy:

$a_{i, k}=\frac{\partial T_{\text {solvus }}\left(R_{k}, X_{1, k}^{\gamma *}, \ldots, X_{N, k}^{\gamma *}\right)}{\partial X_{i}^{\gamma *}}$

$b_{k}=\frac{\partial T_{\text {solvus }}\left(R_{k}, X_{1, k}^{\gamma *}, \ldots, X_{N, k}^{\gamma *}\right)}{\partial \Delta G_{G T}}$

The matrix and precipitate concentrations are related by linearized partition coefficients as follows:

$$
\begin{aligned}
X_{i, k}^{\gamma^{\prime} *} & =k_{i, k}\left(T, R_{k}, X_{1, k}^{\gamma^{*}}, \ldots, X_{N, k}^{\gamma^{*}}\right) X_{i, k}^{\gamma *} \\
& \cong\left[k_{i, k}^{r e f}+\sum_{j=1}^{N} \alpha_{i j, k}\left(X_{j, k}^{\gamma *}-X_{j, k}^{\eta *, r e f}\right)+\beta_{i, k}\left(\Delta G_{G T, k}^{\gamma^{\prime}}-\Delta G_{G T, k}^{\gamma^{\prime}, r e f}\right)\right] \times X_{i, k}^{\gamma *}
\end{aligned}
$$

where $\alpha_{i j, k}$ and $\beta_{i, k}$ are the sensitivity coefficients with respect to the interface concentrations of solute elements $j$ and the curvature energy, respectively: 
$\alpha_{i j, k}=\frac{\partial k_{i, k}\left(R_{k}, X_{1, k}^{\gamma *}, \ldots, X_{N, k}^{\gamma *}\right)}{\partial X_{j}^{\gamma *}}$

$\beta_{i, k}=\frac{\partial k_{i, k}\left(R_{k}, X_{1, k}^{\gamma *}, \ldots, X_{N, k}^{\gamma *}\right)}{\partial \Delta G_{G T}^{\gamma^{\prime}}}$

The reference values for each class $k, T_{\text {solvus }, k}^{\text {ref }}, X_{i, k}^{\gamma *, r e f}$, $\Delta G_{G T, k}^{\gamma^{\prime}}$ and $k_{i, k}^{r e f}$, are computed by calling Thermo-Calc using the current temperature $T$ and the curvature energy contribution corresponding to the class radius. The partial derivatives, $\alpha_{i j, k}, \beta_{i, k}, a_{i, k}$ and $b_{k}$, are obtained by a series of equilibrium calculations using perturbed values of the interface concentrations and of the curvature energy.

In the equilibrium cell previously defined, the average concentrations can be expressed, for each size class, as follows:

$\bar{X}_{i, k}^{\text {cell }}=\left[\left(1-\varphi_{k}^{\gamma^{\prime}}\right)+\varphi_{k}^{\gamma^{\prime}} k_{i}\right] X_{i}^{\gamma^{*}}$

Combining with Eqs. (31) and (34), the $2 N+1$ unknowns $X_{i, k}^{\gamma *}, X_{i, k}^{\gamma^{\prime} *}$ and $\varphi_{k}^{\gamma^{\prime}}$ can now be determined. This set of equations is solved with the Newton-Raphson numerical method. The linearized phase diagram representation is used as long as the two following criteria are satisfied:

$\max _{i}\left(\frac{\left|X_{i, k}^{\gamma *}-X_{i, k}^{\gamma * r e f}\right|}{X_{0, i}}\right)<\varepsilon_{X}^{l i n}$

$\frac{\left|\Delta G_{G T, k}-\Delta G_{G T, k}^{r e f}\right|}{\Delta G_{G T, k}}<\varepsilon_{\Delta G}^{l i n}$

where $\varepsilon_{X}^{l i n}$ and $\varepsilon_{\Delta G}^{l i n}$ are parameters arbitrarily fixed to $1 \%$. If one of the conditions is not fulfilled, the reference values and sensitivity coefficients of the solvus are updated by direct equilibrium computations, as described in the previous paragraph. The above optimized coupling allows for a substantial reduction of the CPU time.

\section{Results and discussion}

\subsection{Simulations of isothermal ageing of $\mathrm{Ni}-7.56 \mathrm{at} \% \mathrm{Al}-$ 8.56 at. $\% \mathrm{Cr}$}

The model was used to simulate isothermal heat treatment of $\mathrm{Ni}-7.56$ at. $\% \mathrm{Al}-8.56$ at. $\% \mathrm{Cr}$ at $600{ }^{\circ} \mathrm{C}$ during $4 \times 10^{6} \mathrm{~s}$. These conditions correspond to those studied by Booth-Morrison et al. [19] and Mao et al. [27] for the atom probe tomography characterization of $\gamma^{\prime}$ precipitates in $\mathrm{Ni}-\mathrm{Al}-\mathrm{Cr}$ alloys. Initially, the alloy was assumed to be free of $\gamma^{\prime}$ precipitates. The diffusion matrix used in the simulation is given in Table 1. The values were calculated from data published by Mao et al. [27], which are based on Monte Carlo simulations of the diffusion-driven growth of $\gamma^{\prime}$ precipitates. The other input parameters are summarized in Table 2. Nucleation was assumed to be homogeneous, with a number density of potential nucleation
Table 1

Diffusion coefficients $\left(\mathrm{m}^{2} \mathrm{~s}^{-1}\right)$ in the $\gamma$ matrix used to simulate precipitation in $\mathrm{Ni}-7.56$ at. $\% \mathrm{Al}-8.56$ at. $\% \mathrm{Cr}$ at $600{ }^{\circ} \mathrm{C}$ (calculated from Ref. [27]).

\begin{tabular}{llll}
\hline$D_{A l, A l}^{\gamma}$ & $D_{A l, C r}^{\gamma}$ & $D_{C r, A l}^{\gamma}$ & $D_{C r, C r}^{\gamma}$ \\
\hline $2.08 \times 10^{-20}$ & $8.59 \times 10^{-21}$ & $8.13 \times 10^{-21}$ & $3.82 \times 10^{-21}$ \\
\hline
\end{tabular}

Table 2

Input parameters of the precipitation model used for the full simulations.

\begin{tabular}{lll}
\hline$\sigma^{\gamma / \gamma^{\prime}}$ & $V_{m}$ & $N_{o}$ \\
\hline $0.027 \mathrm{~J} \mathrm{~m}^{-2}[19]$ & $6.8 \times 10^{-6} \mathrm{~m}^{3} \mathrm{~mol}^{-1}$ & $8.9 \times 10^{28} \mathrm{~m}^{-3}$ \\
\hline
\end{tabular}

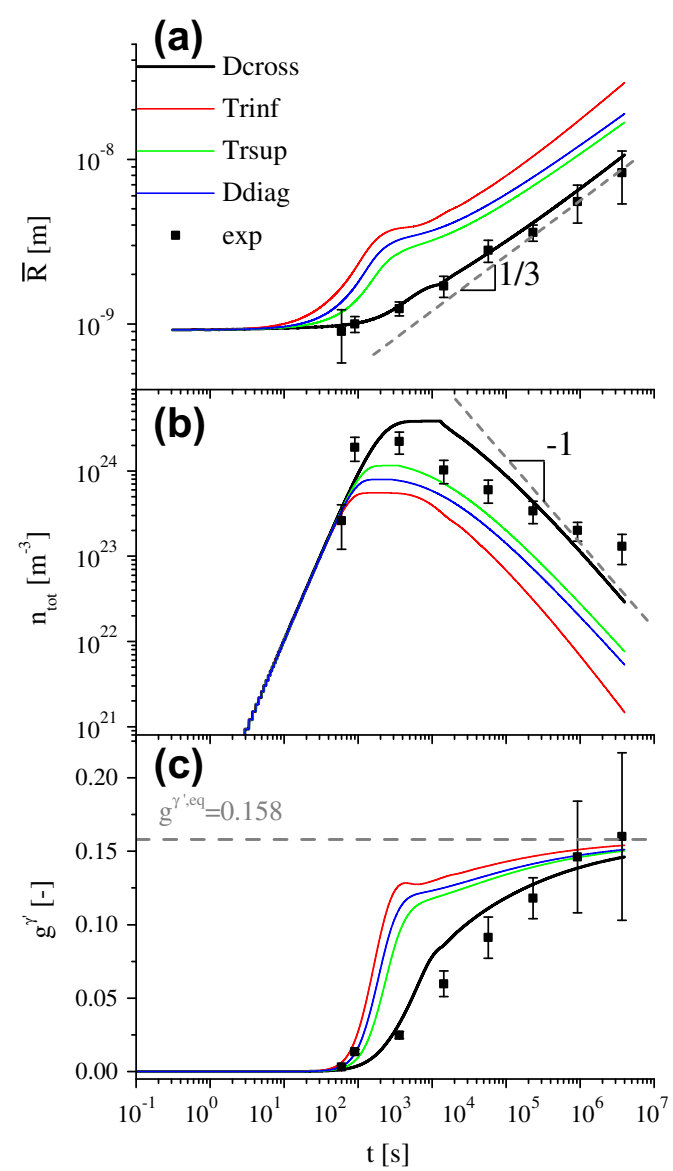

Fig. 2. Time evolution of (a) the mean precipitate radius, $\bar{R}$, (b) the total number density, $n_{\text {tot }}$ and (c) the volume fraction of the $\gamma^{\prime}$ precipitates, $g^{\gamma^{\prime}}$, in heat-treated $\mathrm{Ni}-7.56$ at. $\% \mathrm{Al}-8.56$ at. $\% \mathrm{Cr}$ at $600{ }^{\circ} \mathrm{C}$. The symbols correspond to the experimental data of Booth-Morrison et al. [19].

sites, $n_{0}=N_{a v} / V_{m}$, where $N_{a v}$ is the Avogadro number $\left(\mathrm{mol}^{-1}\right)$ and $V_{m}$ is the constant molar volume $\left(\mathrm{m}^{3} \mathrm{~mol}^{-1}\right)$.

The evolution of $\bar{R}, n_{t o t}$, and $g^{\gamma^{\prime}}$ is shown in Fig. 2 (thick black curves). During the first regime $(t<200 \mathrm{~s})$, the number density of precipitates quickly increases, whereas almost no change of the average radius can be noticed. The $\gamma^{\prime}$ fraction remains close to 0 due to the small size of the precipitates. This regime corresponds to the nucleation of $\gamma^{\prime}$ precipitates. As shown in Fig. 2b, growth occurs essentially in the time interval $200 \mathrm{~s}<t<10^{4} \mathrm{~s}$, during which the 
nucleation rate diminishes due to the decrease of the supersaturation of the matrix and thus the nucleation driving force. During this regime, the volume fraction of precipitates increases due to the diffusion-controlled growth and nucleation (Fig. 2c). At about $t=10^{4} \mathrm{~s}$ the maximal number density is reached, and growth is the only mechanism contributing to the increase of $g^{\gamma^{\prime}}$. As the supersaturation of $\gamma$ decreases, curvature effects have a larger influence on the evolution of the precipitate sizes, number density and volume fraction. At longer ageing times, coarsening becomes dominant, which is characterized by a very slow increase of the volume fraction, while the averaged radius keeps increasing. The time dependencies of $\bar{R}$ and $n_{t o t}$ are close to $t^{1 / 3}$ and $t^{-1}$, respectively, as predicted by the classical LSW theory. This is illustrated by the dashed grey curves in Fig. $2 a$ and $b$.

Fig. 3 shows the cumulative size distributions at different ageing times. The maximum value of the cumulated distribution increases from the initial stage to $10^{4} \mathrm{~s}$, due to the nucleation. Simultaneously, the distributions are shifted to higher precipitate sizes without significant changes of shape, this being determined by the growth of the nucleated precipitates. At $1.3 \times 10^{4} \mathrm{~s}$, the maximum density is the same as at $10^{4} \mathrm{~s}$, but the radius of the smallest precipitates is less. This can be explained by the dissolution of the smallest precipitates. For longer ageing times, broadening of the distribution and decrease of the maximum value occur simultaneously, which corresponds to a mixed growth/coarsening regime with an increasing influence of coarsening as $g^{\gamma^{\prime}}$ approaches the equilibrium value. This can also be seen in Fig. 4, where the normalized size distributions are shown at different times. At $t=1.3 \times 10^{4} \mathrm{~s}$, the dissolution of the smallest precipitates is not completely achieved. However, the shape of the corresponding cumulated distribution indicates a very fast shrinking, which will lead to the total dissolution of the smallest classes and cause the decrease of the total precipitate density observed in Figs. $2 \mathrm{~b}$ and 3. After $10^{6} \mathrm{~s}$, the shape of the normalized distributions remains similar, with a single maximum corresponding to a normalized radius of $\sim 1.2$. One can

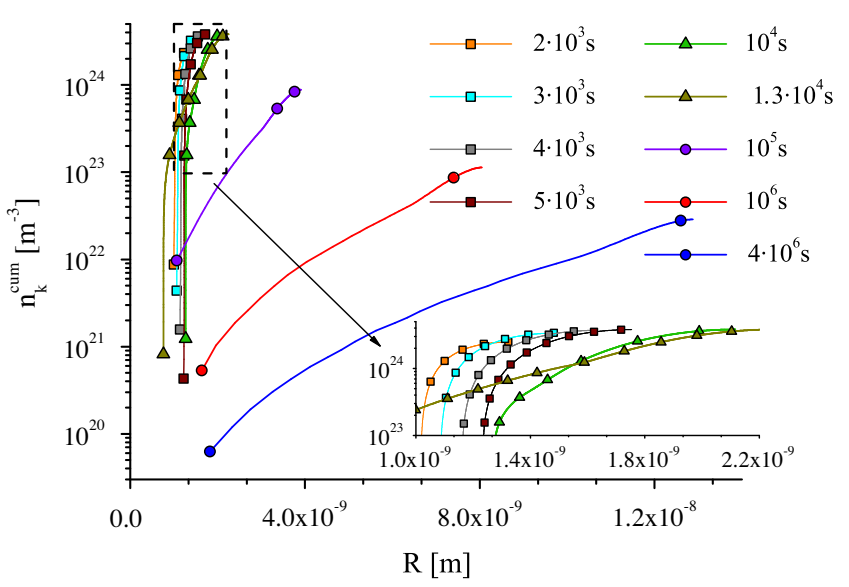

Fig. 3. Calculated cumulative size distributions at 2000 s, 3000 s, 4000 s, $5000 \mathrm{~s}, 10^{4} \mathrm{~s}, 1.3 \times 10^{4} \mathrm{~s}, 10^{5} \mathrm{~s}$ and $4 \times 10^{6} \mathrm{~s}$.

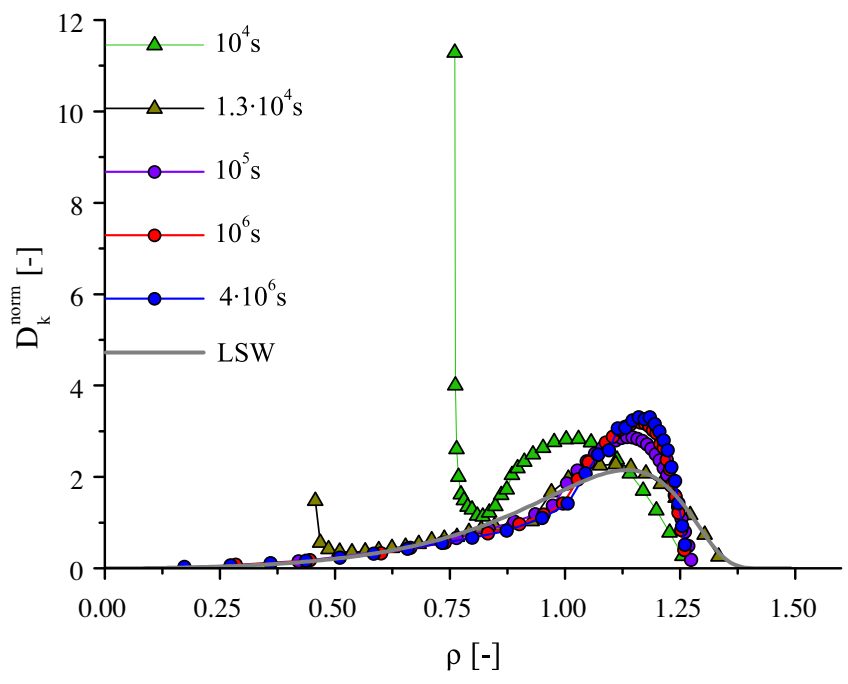

Fig. 4. Normalized size distributions, $D_{k}^{\text {norm }}$, vs. normalized radius, $\rho$, at $10^{4} \mathrm{~s}, 1.3 \times 10^{4} \mathrm{~s}, 10^{5} \mathrm{~s}, 10^{6} \mathrm{~s}$ and $4 \times 10^{6} \mathrm{~s}$ (lines with symbols) and theoretical LSW distribution.

observe in Fig. 4 that a steady state has been reached after $10^{6} \mathrm{~s}$. The final normalized distribution does not superimpose exactly with the theoretical Lifshitz-Slyozov-Wagner (LSW) distribution also plotted in Fig. 4. This could indicate that coarsening is not the only operating phenomenon. Another explanation could be that the assumptions of the LSW theory do not apply exactly here, due to the residual supersaturation of $\gamma$ and the complex dependency of interface concentrations with respect to the radius of the $\gamma^{\prime}$ precipitates. Indeed, the LSW theory was formulated for binary alloys with the approximation of an ideal dilute solution, which is not the case for the numerical model. Finally, the cross-diffusion can have a strong influence on the growth/dissolution kinetics, which may also result in differences with the theoretical distribution. This aspect will be analysed in more detail in the next paragraphs.

\subsection{Comparisons with experimental data}

The experimental data published by Booth-Morrison et al. [19] have been reported in Figs. 2 and 5. As can be seen in Fig. 2a, the average precipitate radii predicted by the model (black curve labelled "Dcross") are in good agreement with the measured values. The trends are also similar for the total number density $n_{t o t}$, although in the simulation the maximal value of the precipitate density is higher than in the experiments (Fig. 2b). These discrepancies can be explained by the assumption of homogeneous nucleation. One can note that the differences in $g^{\gamma^{\prime}}$ reflect those observed for $n_{t o t}$.

Fig. 5 shows the paths of the simulated and measured concentrations in $\gamma$ and $\gamma^{\prime}$ during precipitation. In the simulation, the final average concentrations, labelled (e), lie on the equilibrium tie-line given by the thermodynamics database [28]. Measurements are also found close to the equilibrium tie-line at points (e), but at different positions compared with the simulation. In $\gamma^{\prime}$, important discrepancies between 


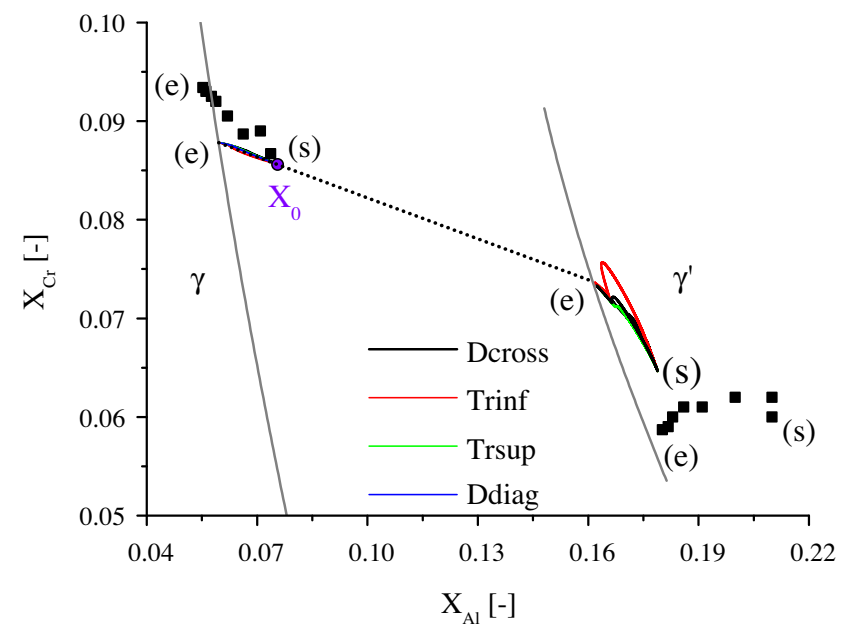

Fig. 5. Evolution of average concentrations in the $\gamma$ matrix and in the $\gamma^{\prime}$ precipitates during heat treatment of $\mathrm{Ni}-7.56 \mathrm{at} . \% \mathrm{Al}-8.56 \mathrm{at} . \% \mathrm{Cr}$ at $600{ }^{\circ} \mathrm{C}$. The symbols correspond to the experimental data of BoothMorrison et al. [19]. Simulation results are shown with continuous lines. The $\gamma$ and $\gamma^{\prime}$ solvuses were obtained from the Ni20 thermodynamic database without considering the Gibbs-Thomson effect (continuous grey lines). The equilibrium tie-line is indicated with the black dotted line. $\mathrm{X}_{0}$ indicates the nominal composition of the alloy.

calculated and measured concentrations are observed. The initial $\mathrm{Al}$ concentrations, labelled (s), are significantly lower in the simulation than in the measurements (by $\sim 3$ at.\%). Also, the model does not predict the global decrease of the average $\mathrm{Cr}$ concentration in $\gamma^{\prime}$ observed experimentally.

A possible source of discrepancies between simulation and experiments is the mean field approach used for the concentration fields, which does not take into account the local shapes of the concentration profiles influenced by the high particle density and the relative positions of the precipitates. In the model, coarsening is assumed to occur only via the diffusion fluxes in the matrix, whereas in real systems, direct contacts between $\gamma^{\prime}$ precipitates can occur and lead to coalescence or coagulation events, as reported in Ref. [19]. Substantial differences are observed on the equilibrium concentrations, which are directly linked to the thermodynamic database [28]. Finally, the role of vacancies, which may have a strong influence on the diffusion kinetics and possibly also on nucleation, is not accounted for in the simulation. In spite of these limitations, the overall transformation kinetics (average radius and volume fraction of precipitates) are well reproduced by the model. It is worth mentioning that the results of the simulation were obtained with the assumption of homogeneous nucleation, which, unlike heterogeneous nucleation laws, does not involve adjustable parameters. The interfacial energy used in the simulation, $27 \mathrm{~mJ} \mathrm{~m}^{-2}$, lies in the experimental range given by Booth-Morrison et al. $\left(24 \pm 6 \mathrm{~mJ} \mathrm{~m}^{-2}\right)$ [19].

\subsection{Importance of cross-diffusion}

The role of the non-diagonal diffusion coefficients on the precipitation response of the alloy has been analysed in more detail by performing calculations with different diffu- sion matrices. Three new cases were considered. They correspond to simplified versions of the diffusion matrix shown in Table 1. In the case which will be referred to as "Trinf", the term $D_{A l, C r}^{\gamma}$ was set to 0 , whereas in the case "Trsup", $D_{C r, A l}^{\gamma}$ was set to 0 . The case referred to as "Diag" corresponds to the diagonal matrix where both non-diagonal terms are ignored: $D_{A l, C r}^{\gamma}=D_{C r . A l}^{\gamma}=0$. The diagonal coefficients were always those given in Table 1 .

The results obtained for the three new cases are reported in Fig. 2, where they are compared with the results for the full diffusion matrix ("Dcross") already presented in the previous paragraph. The overall behaviour is qualitatively similar for all cases. The nucleation, growth and coarsening regimes discussed in the previous paragraph can also be observed for the simplified diffusion matrices. However, the results are substantially different from a quantitative point of view. The quickest growth kinetics is observed for the Trinf case. The $\gamma^{\prime}$ fraction increases faster for this case, and thus the nucleation driving force also decreases earlier. Consequently, the total precipitate density, $n_{\text {tot }}$ (Fig. 2b), is the lowest. The slowest precipitation kinetics among the four cases is observed for Dcross. The nucleation rate remains high during a long period of time, which leads to the highest maximum for $n_{\text {tot }}$. Intermediate behaviours are observed for the Diag and Trsup cases.

To better isolate the role of each diffusion coefficient, an independent analysis has been conducted. The model was applied to the case of a single precipitate growing in a spherical volume of matrix. The initial precipitate radius was $10^{-9} \mathrm{~m}$, and the radius of computation domain was $10.6078 \mathrm{~nm}$, corresponding to a number density of $2 \times 10^{23} \mathrm{~m}^{-3}$. No Gibbs-Thomson effect was considered. As for the first type of simulations, the temperature was kept constant, at $600{ }^{\circ} \mathrm{C}$. The calculations were carried out for the four diffusion matrices, Dcross, Trsup, Trinf and Diag. In addition the Diag calculation was repeated using a frozen concentration profile in the precipitate, rather than full mixing, which is similar to the GulliverScheil approximation. This case will be referred hereafter as "Diag-Scheil".

Fig. 6 shows the evolution of the radius for the five cases. As for the multiclass simulations, the highest growth rate is observed for the lower triangular matrix (Trinf) and the slowest for the full diffusion matrix (Dcross). Between these two extrema, the Diag case exhibits slightly higher growth rates than Trsup. These variations are similar to those for the overall kinetics displayed in Fig. 2.

As shown in Fig. 5, $\gamma^{\prime}$ is richer in $\mathrm{Al}$ and poorer in $\mathrm{Cr}$ than $\gamma$. As growth proceeds, the matrix is progressively depleted in $\mathrm{Al}$ and enriched in $\mathrm{Cr}$, and the two profiles have opposite variations. Therefore, the non-diagonal coefficients of the matrix, which are both positive, tend to decrease the magnitude of the $\mathrm{Al}$ and $\mathrm{Cr}$ fluxes computed with Eq. (7). This effect explains qualitatively why the Dcross case has the slowest kinetics, and why Trsup has a slightly slower kinetics than Diag. However, the fact that Trinf exhibits faster growth kinetics than Diag shows that 


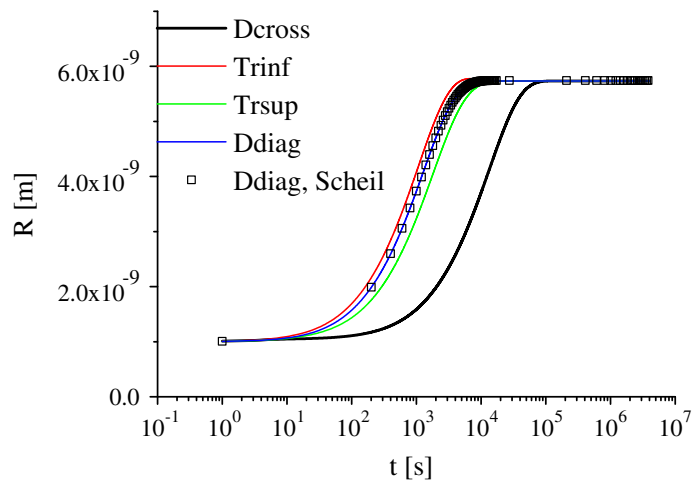

Fig. 6. Variations of the radius with time, for the four diffusion matrices Diag, Trinf, Trsup and Cross. For the case Diag, two hypotheses were taken into account for the concentration profiles in $\gamma^{\prime}$ : complete mixing (standard case, full blue line) and Scheil (squares). (For interpretation of the references to color in this figure legend, the reader is referred to the web version of this article.)

this argument can be misleading. The variations of the diffusion fluxes and the modification of the tie-line during the transformation have to be analysed more carefully to understand the influence of the diffusion matrix.

Fig. 7 shows the composition paths in the $\gamma$ phase for the four cases. The deviation of the trajectory from the equilibrium tie-line indicates that the $\mathrm{Al}$ depletion of the matrix occurs faster than the $\mathrm{Cr}$ enrichment. This effect is more pronounced for Trinf and Dcross but also visible for the other cases. It is obviously associated with the high value of the $D_{A l, A l}^{\gamma}$ diffusion coefficient reported in Table 1 .

Figs. 8-10 show the evolution of the average concentrations, interface concentrations and solute fluxes in $\gamma$ at the $\gamma / \gamma^{\prime}$ interface. It can be seen in Fig. 10 that for Trinf and Dcross the Cr flux is negative at the beginning of the growth $(t<6 \mathrm{~s})$. This is at first sight unexpected, knowing that $X_{C r}^{\gamma *}>X_{0, C r}$, but it can be easily understood by considering the high value of $D_{C r . A l}^{\gamma}$, which induces a negative cross-contribution of larger magnitude than the diagonal one. Because $X_{C r}^{\gamma^{\prime *}}<X_{C r}^{\gamma *}$, a negative flux tends to dissolve the precipitate and/or modify the operating tie-line towards

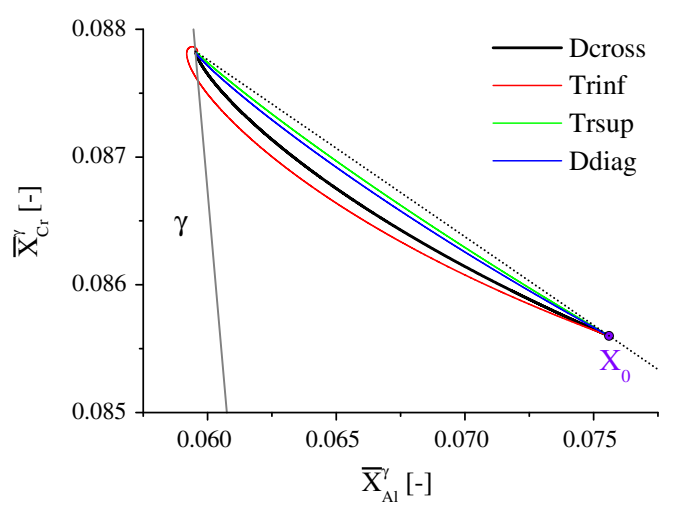

Fig. 7. Evolution of the average concentrations in the $\gamma$ phase during the growth sequence shown in Fig. 6. The solvus of $\gamma$ and the equilibrium tieline are indicated by the grey and dotted black lines, respectively.

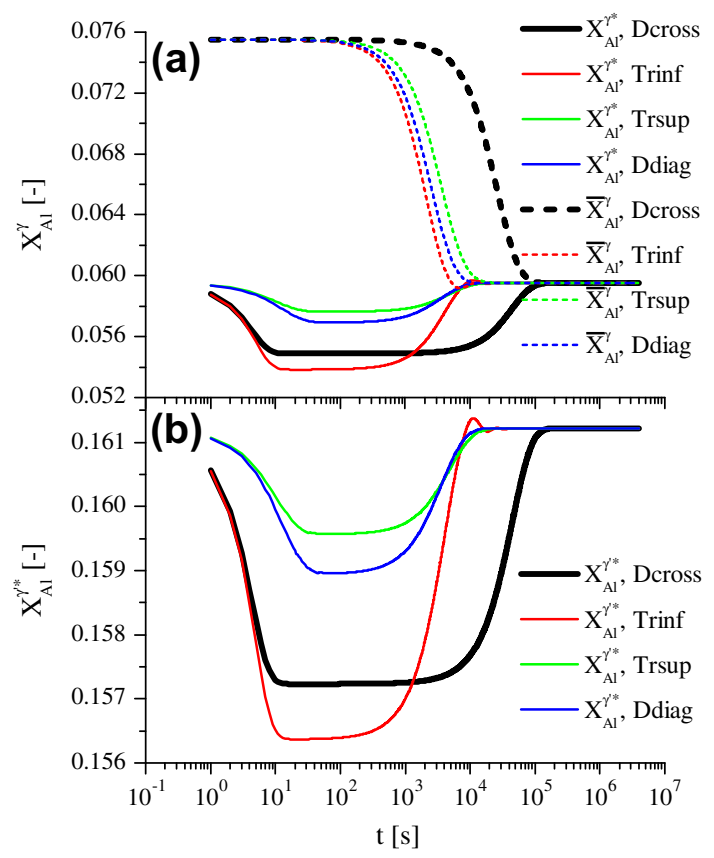

Fig. 8. Variations of the $\mathrm{Al}$ concentrations in (a) $\gamma$ and (b) $\gamma^{\prime}$ for the four diffusion matrices Diag, Trinf, Trsup and Dcross. $X_{A l}^{\gamma *}$ and $\bar{X}_{A l}^{\gamma}$ are the interface and the average concentration in $\gamma$, respectively.

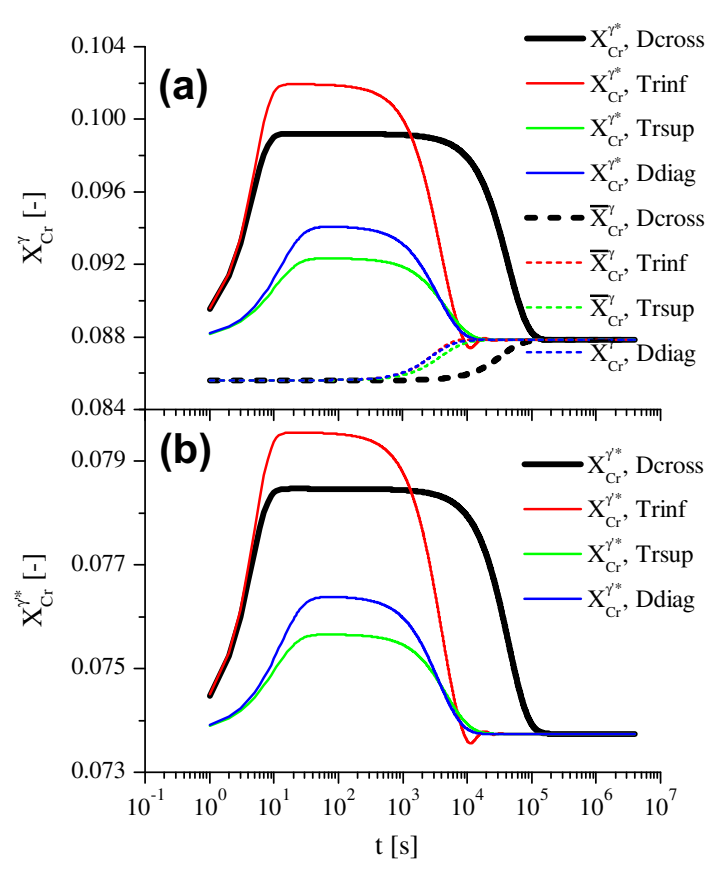

Fig. 9. Variations of the $\mathrm{Cr}$ concentrations in (a) $\gamma$ and (b) $\gamma^{\prime}$ for the four diffusion matrices Diag, Trinf, Trsup and Dcross. $X_{C r}^{\gamma *}$ and $\bar{X}_{C r}^{\gamma}$ are the interface and the average concentration in $\gamma$, respectively.

higher values of $X_{C r}^{\gamma *}$ and $X_{C r}^{\gamma^{\prime *}}$. In this case, only the second mechanism occurs because dissolution is not compatible with the negative flux of Al. A fast increase of $X_{C r}^{\gamma *}$ and $X_{C r}^{\gamma^{*}}$ is thus observed (see Fig. 9). At $t>6 \mathrm{~s}, X_{C r}^{\gamma *}$ has sufficiently risen to obtain a positive $\mathrm{Cr}$ flux. The $\mathrm{Cr}$ gradient is then larger than for the Ddiag and Trsup cases. 


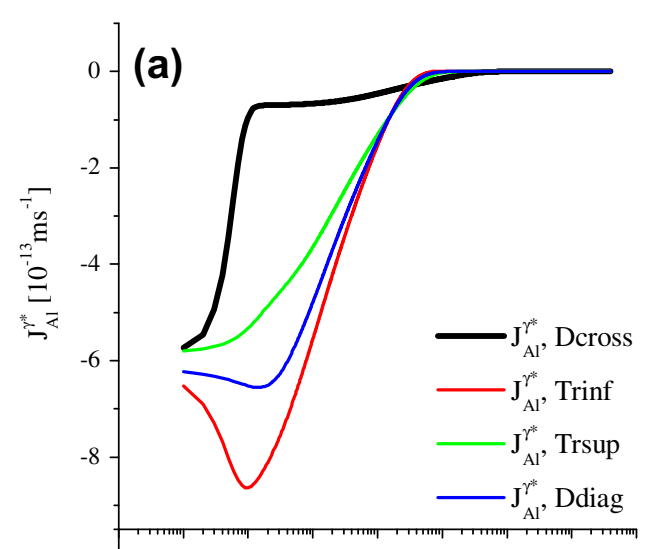

(b)

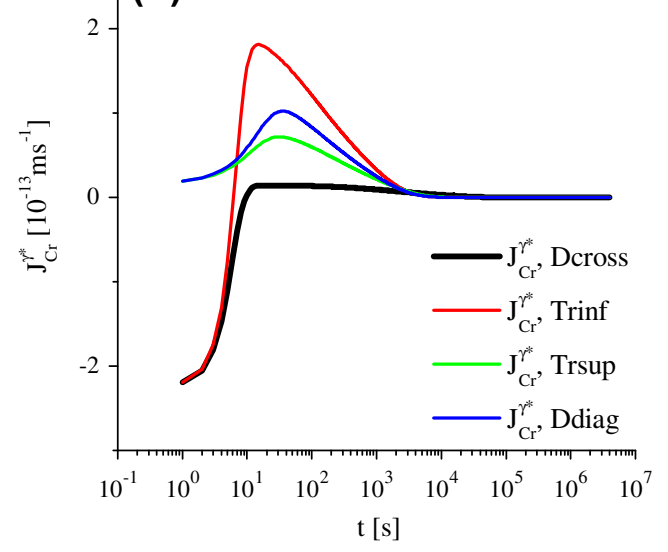

Fig. 10. Variations of the solute flux with time, for (a) $\mathrm{Al}$ and (b) $\mathrm{Cr}$.

In other words, the high value of $D_{C r, A l}^{\gamma}$ brings $C r$ to the interface and helps to establish a steep $\mathrm{Cr}$ gradient in the matrix. This phenomenon tends to enhance the growth in a later stage of the precipitation, when $\mathrm{Al}$ is already well mixed in the matrix. This sequence of phenomena explains the rapid kinetics of Trinf. If both $D_{C r, A l}^{\gamma}$ and $D_{A l, C r}^{\gamma}$ are considered (Dcross), the steep gradient of $\mathrm{Cr}$ will have a negative contribution on the Al flux. More time will be needed to homogenize $\mathrm{Al}$ in the matrix than for the other cases, which translates as a long plateau of $\bar{X}_{A l}^{\gamma} \cong 0.055$ (see Fig. 8). The total precipitation time is finally larger for Dcross than for the other cases.

Fig. 6 shows that the time evolution of the radius is very similar for the complete mixing in the precipitates (Diag) and no diffusion (Diag-Scheil). Due to the relatively small size of the $\gamma^{\prime}$ precipitates, the concentration gradients in $\gamma^{\prime}$ do not significantly influence the overall solute balance, and finally the growth kinetics remains almost unchanged.

\section{Conclusions}

A comprehensive model has been developed for the simulation of $\gamma^{\prime}$ precipitation in a multicomponent $\mathrm{Ni}$ alloy. It is shown that the model can describe the different regimes of precipitation: nucleation, growth and coarsening. After a transient regime between growth and coarsening, during which the particle size distributions rearrange, the present model recovers stationary size distributions and the time dependencies of the average quantities predicted by the LSW theory. The results of the simulation for ageing of $\mathrm{Ni}-7.56$ at. $\%$ Al-8.56 at. $\% \mathrm{Cr}$ at $600{ }^{\circ} \mathrm{C}$ reaches global agreement with the experimental observations by BoothMorrison et al. [19]. The computed evolutions of the phase fraction and average radius are quantitatively close to the measurements. Discrepancies were, however, observed for the nucleation kinetics and the equilibrium concentrations at the $\gamma / \gamma^{\prime}$ interface. A possible reason is the assumption of homogeneous nucleation, which is rather restrictive and does not contain any adjustable parameters to represent the influence of defects such as vacancies or dislocations. Also, the mean-field approach used in this work does not allow for the description of geometrical aspects, such as the precipitate shape and direct contacts between particles. However, the results obtained in this study indicate that the quality of the thermodynamic and kinetic data could be the most limiting factor for more quantitative simulations of precipitation in $\mathrm{Ni}-\mathrm{Al}-\mathrm{Cr}$, especially at low temperatures.

Cross-diffusion (non-diagonal diffusion matrices) turned out to affect significantly the calculated evolution of average concentrations and, to a lower extent, the average radius, number and volume fraction of precipitates. In industrial nickel-base superalloys, this aspect is expected to have an even larger influence on the overall precipitation kinetics, due to the higher solute contents. More generally, the present model offers excellent perspectives to simulate and optimize heat treatment in industrial multicomponent alloys, for which the exploitation of the latest kinetic and thermodynamic databases is crucial for quantitative predictions.

\section{Acknowledgements}

This financial support of the Snecma-SAFRAN group is greatly acknowledged. The authors are also grateful to Prof. M. Rappaz for the fruitful discussions and comments on this subject.

\section{References}

[1] Reed RC. The superalloys, fundamentals and applications. Cambridge, UK: Cambridge University Press; 2006.

[2] Lifshitz IM, Slyozov VV. J Phys Chem Solids 1961;19:35.

[3] Langer JS, Schwartz AJ. Phys Rev A 1980;21:948.

[4] Kampmann R, Wagner R. In: Haasen P, Gerold V, Wagner R, Ashby Michael F, editors. Decomposition of alloys: the early stages. Sonnenberg: Pergamon; 1984. p. 91.

[5] Robson JD, Prangnell PB. Acta Mater 2001;49:599.

[6] Nicolas M, Deschamps A. Acta Mater 2003;51:6077.

[7] Serriere M, Gandin CA, Gautier E, Archambault P, Dehmas M. Mater Sci Forum 2002;396-402:747.

[8] Chen Q, Jeppsson J, Agren J. Acta Mater 2008;56:1890.

[9] Svoboda J, Fischer FD, Fratzl P, Kozeschnik E. Mater Sci Eng A 2004;385:166.

[10] Kozeschnik E, Svoboda J, Fratzl P, Fischer FD. Mater Sci Eng A 2004;385:157.

[11] Pareige C, Soisson F, Martin G, Blavette D. Acta Mater 1999;47:1889. 
[12] Guyot P, Sigli C. In: Poole WJ, Wells MA, Lloyd DJ, editors. Aluminium alloys 2006, Pts 1 and 2: research through innovation and technology, vol. vols. 519-521. Zurich-Uetikon: Trans Tech; 2006. p. 291.

[13] Bratland DH, Grong Ø, Shercliff H, Myhr OR, Tjøtta S. Acta Mater 1997;45:1.

[14] Gandin CA, Jacot A. Acta Mater 2007;55:2539.

[15] Gandin CA, Bréchet Y, Rappaz M, Canova G, Ashby M, Shercliff H. Acta Mater 2002;50:901.

[16] Du Q, Perez M, Poole WJ, Wells M. Scripta Mater 2012;66:419.

[17] Du Q, Poole WJ, Wells MA. Acta Mater 2012;60:3830.

[18] Campbell CE, Boettinger WJ, Kattner UR. Acta Mater 2002; 50:775.
[19] Booth-Morrison C, Weninger J, Sudbrack CK, Mao Z, Noebe RD, Seidman DN. Acta Mater 2008;56:3422.

[20] Perez M, Dumont M, Acevedo-Reyes D. Acta Mater 2008;56:2119.

[21] Myhr OR, Grong Ø. Acta Mater 2000;48:1605.

[22] Russel KC. Adv Colloid Interface Sci 1980;13:215.

[23] Aaronson HI, Lee JK.In: Aaronson H.I., editors. Lectures on the Theory of Phase Transformations, TMS-AIME. New York, 1975.

[24] Maugis P, Gouné M. Acta Mater 2005;53:3359.

[25] Aaron H, Fainstein D, Kotler G. J Appl Phys 1970;41:4404.

[26] Jacot A, Rappaz M. Acta Mater 2002;50:1909.

[27] Langer Z, Booth-Morrison C, Sudbrack CK, Martin G, Seidman DN. Acta Mater 2012;60:1871.

[28] Ni20: Ni Base Alloys Database 2010. Thermo-Calc Software AB. 